\title{
ORIGINAL
}

\section{Pantoprazole prophylaxis in ICU patients with high severity of disease: a post hoc analysis of the placebo-controlled SUP-ICU trial}

\author{
Søren Marker ${ }^{1,2^{*}}$ (อ, Anders Perner ${ }^{1,2}$, Jørn Wetterslev ${ }^{2,3}$, Mette Krag ${ }^{1,2}$, Theis Lange ${ }^{4,5}$, Matt P. Wise ${ }^{6}$, \\ Mark Borthwick ${ }^{7}$, Stepani Bendel ${ }^{8}$, Frederik Keus ${ }^{9}$, Anne Berit Guttormsen ${ }^{10,11}$, Joerg C. Schefold ${ }^{12}$, \\ Morten Hylander Møller ${ }^{1,2}$ and The SUP-ICU investigators
}

(c) 2019 Springer-Verlag GmbH Germany, part of Springer Nature

\begin{abstract}
Purpose: In the subgroup of patients with Simplified Acute Physiology Score (SAPS) II > 53 in the Stress Ulcer Prophylaxis in Intensive Care Unit (SUP-ICU) trial, there was interaction $(P=0.049)$ suggesting increased mortality in patients allocated to pantoprazole as compared with placebo. We aimed to explore this further.

Methods: The SUP-ICU trial allocated acutely admitted adults at risk of gastrointestinal bleeding to pantoprazole vs placebo. In this post hoc study, we repeated all the preplanned analyses of SUP-ICU in patients with baseline SAPS II $>53$.

Results: A total of 1140 patients had a complete SAPS II $>53$ and were included. At 90 days, 272/579 patients (47\%) assigned to pantoprazole had died, as compared with 229/558 patients (41\%) assigned to placebo [relative risk 1.13; $95 \%$ confidence interval (CI) 1.00-1.29]. This was supported by sensitivity analyses adjusted for risk factors and those in the per-protocol population. When accounting for patients with incomplete SAPS II in two additional analyses, the relative risk was 1.08; 95\% $\mathrm{Cl} 0.96-1.22$ and $1.10 ; 95 \% \mathrm{Cl} 0.97-1.25$. This was also observed for the secondary outcome days alive without life support. There were no differences between the intervention groups in the other secondary outcomes.
\end{abstract}

Conclusions: In this post hoc analysis of patients with high disease severity included in the SUP-ICU trial, we observed higher 90-day mortality and fewer days alive without life support with pantoprazole vs placebo. Some of this may have been explained by missing SAPS II data, but further research is needed to draw firm conclusions.

ClinicalTrials.gov: ClinicalTrials.gov No. NCT02467621.

Keywords: Stress ulcer prophylaxis, Stress ulceration, Intensive care unit, Gastrointestinal bleeding, All-cause mortality

\footnotetext{
*Correspondence: soeren.marker@regionh.dk

${ }^{1}$ Department of Intensive Care 4131, Copenhagen University Hospital,

Rigshospitalet, Blegdamsvej 9, 2100 Copenhagen, Denmark

Full author information is available at the end of the article
}

\section{县




\section{Introduction}

Critically ill patients in the intensive care unit (ICU) are at risk of developing clinically important gastrointestinal (GI) bleeding due to stress ulcers [1-3]. GI bleeding in critically ill patients has been suggested to be associated with adverse outcomes, including a 2-4-fold increased risk of death and increased length of ICU stay [3]. To prevent GI bleeding, stress ulcer prophylaxis is currently recommended in international guidelines [4] and acid suppressants are frequently used in ICU patients $[2,5]$.

In the international Stress Ulcer Prophylaxis in the Intensive Care Unit (SUP-ICU) trial, 3350 patients at risk of GI bleeding were randomised to receive either pantoprazole or placebo during the ICU stay [1]. We observed no difference between the pantoprazole and placebo groups in the primary outcome measure-90-day mortality-but a predefined subgroup analysis suggested higher 90-day mortality in the pantoprazole group among patients who had higher baseline disease severity, i.e., Simplified Acute Physiology Score (SAPS) II $>53$ points (test of interaction, $p$ value 0.049 ) [6,7]. This warrants further investigation, because it may be a chance finding or driven by baseline imbalance or differences in withdrawals or follow-up in the subgroup, or missing data for SAPS II elements in some patients.

In this post hoc study of the SUP-ICU trial, we performed the full set of preplanned outcome analyses in all patients with baseline SAPS II $>53$ allocated to pantoprazole or placebo on all trial outcomes to search for explanations to substantiate or refute the suggested harm from pantoprazole in the most severely ill. We hypothesised that the increased 90-day mortality observed in patients with SAPS II $>53$ allocated to pantoprazole would be substantiated in sensitivity analyses and in those of the remaining outcomes.

\section{Methods}

\section{Study oversight}

This study is a post hoc analysis of the SUP-ICU trial [7]. The trial protocol, statistical analysis plan and primary results have been published elsewhere $[1,7,8]$. The SUP-ICU trial was approved by all relevant institutions prior to randomisation of the first patient. No additional approvals were required for this study. We have prepared this manuscript according to the Strengthening the Reporting of Observational Studies in Epidemiology (STROBE) statement [9], and the filled-in checklist is available in the Electronic Supplementary Material (ESM).

\section{Take-home message}

In this post-hoc analysis of a predefined subgroup population in the Stress Ulcer Prophylaxis in the Intensive Care Unit (SUP-ICU) trial, we observed higher 90-day mortality and fewer days alive without the use of life support in patients with a complete Simplified Acute Physiology Score (SAPS) II > 53 points allocated to pantoprazole. We found no explanations for these observations in baseline data, process variables or the other trial outcome measures-however, missing SAPS II data for some patients seem to explain a part of the observed difference.

\section{The SUP-ICU trial}

The SUP-ICU trial was a randomised, multicenter, stratified, parallel-group, placebo-controlled, blinded clinical trial recruiting 3350 patients (3291 were available for data analyses) at risk for GI bleeding in 33 ICUs in Denmark, Finland, Norway, Switzerland, the Netherlands and UK from 4 January 2016 to 22 October 2017 [7]. Eligible subjects were patients aged 18 years or older, who were acutely admitted to the ICU and had at least one risk factor for clinically important GI bleeding, including any history of liver disease, any history of or ongoing coagulopathy, shock, or use of anticoagulant agents, renal replacement therapy, or mechanical ventilation expected to last $>24 \mathrm{~h}$ [1].

We excluded patients with GI bleeding during current hospital admission, ongoing daily treatment with acid suppressants, patients who were withdrawn from active treatment or brain dead, had organ transplantation during current hospital admission, had peptic ulcer confirmed by endoscopy or other method during current hospital admission, had a contraindication to pantoprazole, were pregnant or where consent for enrolment could not be obtained. The full definitions of the inclusion and exclusion criteria are presented in the ESM.

Patients were randomly assigned to daily intravenous pantoprazole $40 \mathrm{mg}$ or a matching placebo during the ICU stay. Randomisation was stratified according to the presence or absence of hematological malignancy and trial site. The intervention period lasted until discharge from the ICU to a maximum of 90 days. The protocol was reinitiated in case of readmissions to trial ICUs within this period. Apart from the use of stress ulcer prophylaxis, all other parts of patient care were at the discretion of the clinicians.

At baseline we collected data on demographics and clinical characteristics. Daily recordings during the entire admission to the ICU included GI bleeding events, infectious adverse events (pneumonia and Clostridium difficile infection) and data on the usage of life support 
modalities. Time of death was registered for all patients at a maximum follow-up of 90 days. Observations for GI bleeding and infectious adverse events stopped at the time of discharge from the ICU.

Informed consent was obtained prior to randomisation from all participants or their legal substitutes according to national legislation.

\section{Outcomes}

The primary outcome was 90-day mortality. Secondary outcomes were the proportion of patients with clinically important events (a composite outcome of clinically significant GI bleeding, pneumonia, Clostridium difficile infection and myocardial ischemia), clinically important GI bleeding, infectious adverse events (pneumonia or Clostridium difficile infection) and serious adverse reactions (SARs) and the percentage of days alive without the use of life support (mechanical ventilation, renal replacement therapy or inotropes/vasopressor) [1].

\section{Definitions}

Clinically important GI bleeding was defined as overt GI bleeding and at least one of the following four features within $24 \mathrm{~h}$ of the bleeding episode, in the absence of other causes, in the ICU: (1) a spontaneous drop of systolic blood pressure, mean arterial pressure or diastolic blood pressure of $20 \mathrm{mmHg}$ or more, (2) start of a vasopressor or a $20 \%$ increase in vasopressor dose, (3) decrease in hemoglobin of at least $2 \mathrm{~g} / \mathrm{dl}(1.24 \mathrm{mmol} / \mathrm{l})$, or (4) transfusion of two or more units of packed red blood cells [1]. The full definitions of the other outcomes are presented in the ESM.

\section{Statistical analyses}

We repeated all predefined analyses on all outcomes and in all predefined subgroups according to the published SUP-ICU trial statistical analysis plan [8] in this subgroup of patients with baseline SAPS II $>53$. The SAPS II score is calculated from 17 variables with a total range from 0 to 163 points; higher scores indicating greater severity of disease (Table S7 in the ESM). Fifty-three points was chosen a priori as the cut-off for the subgroup analysis, as it was found to predict a $50 \%$ mortality rate in the original model [6] and similar cut-offs have been used previously [10].

In brief, we conducted the primary analyses in the intention-to-treat population. In the primary analyses, we compared data in the two treatment groups by binary logistic regression analysis adjusted for the stratification variables (trial site and active hematological malignancy); relative risks with 95\% confidence intervals were computed from odds ratios [8]. In a sensitivity analysis, we compared the primary outcome in the per-protocol population (excluding patients having one or more major protocol violations-definition in ESM), and in the originally pre-specified subgroups, defined by the presence or absence of any history of liver disease, the presence or absence of any history of or ongoing coagulopathy, the type of ICU admission (medical versus surgical), the presence or absence of shock and the use or not of mechanical ventilation [8].

In the secondary analyses, we compared all dichotomous outcomes using binary logistic regression analyses adjusted for stratification variables and predefined risk factors at baseline (age, type of admission (medical, elective surgery or emergency surgery) and the Sepsis-related Organ Failure Assessment (SOFA) score (Table S8 in the ESM) assessed in the $24 \mathrm{~h}$ before randomisation). We analysed percentages of days alive without life support in the 90-day period by use of the van Elteren test (adjusted for site only), as the assumptions for Poisson or negative binomial distributions were not met [11]. We performed no adjustment for multiple comparisons and reported results as point estimates with $95 \%$ confidence intervals.

To account for the between-group imbalance in coagulopathy, we conducted a post hoc sensitivity analysis in which we included coagulopathy in the adjusted (secondary) binary logistic regression model.

As the proportion of patients with incomplete SAPS II could be of importance, we conducted the following two post hoc sensitivity analyses (of all outcomes): (1) patients with missing SAPS II who could potentially have had SAPS II > 53 were included (assigning the worst possible SAPS II sub-score, when missing, and then recalculating the total SAPS II). An additional 169/249 patients with missing SAPS II were included in this sensitivity analysis; and (2) patients with missing SAPS II who after computer-generated single-imputation of missing SAPS II sub-scores had a SAPS II > 53 were included. The single-imputation approach was repeated three times to assess random variation and showed consistency. An additional 106/249 patients with missing SAPS II were included in this sensitivity analysis.

In the complete-case population, nine patients were lost to 90-day follow-up in the full trial population, of which three had a SAPS II $>53$. Accordingly, completecase analysis was the main primary outcome analysis. Additional details on handling of missing data, including sensitivity analyses with imputation of SAPS II values are provided in the ESM.

All analyses were performed using SAS software, version 9.4, and $\mathrm{R}$ software, version 3.4.3. 
(See figure on next page.)

Fig. 1 Randomisation and follow-up. The list of reasons for exclusions in the SUP-ICU trial was presented in the main publication of the SUP-ICU trial. In brief, 52 patients were originally excluded immediately after randomisation prior to the first dose of trial medication because they did not meet the inclusion criteria or fulfilled one or more exclusion criteria. A total of seven patients were excluded after randomisation because the patient or their surrogate did not allow the use of the data. The applied dotted-line square marks the population for this post hoc analysis of patients with high baseline disease severity (SAPS II > 53). A total of 249 patients, 134 in the pantoprazole and 115 in the placebo group, had missing data for one or more of the SAPS II elements and therefore an incomplete SAPS II. These were excluded from the main analysis, but they were included in sensitivity analyses with the missing data imputed. Of the 1140 patients with a baseline SAPS $\|>53$, one patient was lost for 90 -day follow-up in the pantoprazole group and two patients in the placebo group

\section{Results}

A total of 1140 patients had complete SAPS II $>53$ at baseline; 580 assigned to pantoprazole and 560 assigned to placebo. Besides excluding patients with SAPS II $\leq 53$, we excluded $249 / 3291$ patients $(7.6 \%)$ with incomplete SAPS II (Fig. 1). Ninety-day vital status was obtained for 1137 of 1140 patients (99.5\%), including 579 in the pantoprazole group and 558 in the placebo group (Fig. 1). Patient characteristics at baseline appeared similar in the two groups, except for coagulopathy (Table 1).

\section{Outcomes}

At 90 days after randomisation, 272 of 579 patients (47.0\%) in the pantoprazole group and 229 of 558 (41.0\%) in the placebo group had died (relative risk, 1.13; 95\% confidence interval 1.00-1.29) (Table 2 and Fig. 2). The results were consistent in the sensitivity analysis adjusted for baseline risk factors (relative risk, 1.14; 95\% confidence interval 0.99-1.30), in the per-protocol population (relative risk, 1.17; 95\% confidence interval 1.01-1.37) (Table 2), and in the post hoc sensitivity analysis with adjustment for coagulopathy at baseline estimates (relative risk, 1.14; 95\% confidence interval 0.99-1.30). In the predefined subgroup analyses, we observed no heterogeneity in the effect of pantoprazole versus placebo on mortality at 90 days (Fig. 2).

Some 146 of 580 patients (25.2\%) in the pantoprazole group, as compared with 147 of 560 patients (26.3\%) in the placebo group, had one or more clinically important events in the ICU (relative risk, 0.93; 95\% confidence interval 0.75-1.16) (Table 2). Fewer patients in the pantoprazole group had clinically important GI bleeding; 19 of $580(3.3 \%)$ and 34 of $560(6.1 \%)$ in the pantoprazole and placebo groups, respectively, (relative risk, 0.49 ; $95 \%$ confidence interval $0.29-0.86$ ). The percentage of days alive without the use of life support was $81 \%$ (interquartile range 9-95) and 85\% (interquartile range 36-96), in the pantoprazole and placebo groups $(P=0.02)$, respectively. The remaining secondary outcomes and the remaining single components of the composite outcome were similar between the two groups (Table 2 and Table S1 in the ESM).
In the two post hoc sensitivity analysis accounting for missing SAPS II values (using a logically imputed and a single-imputed SAPS II > 53 population), 299 of 667 patients $(44.8 \%)$ in the pantoprazole group and 264 of $639(41.3 \%)$ in the placebo group had died (relative risk, 1.08; $95 \%$ confidence interval 0.96-1.22), and 292 of 636 patients $(45.9 \%)$ in the pantoprazole group and 253 of $607(41.7 \%)$ in the placebo group had died (relative risk, 1.10; 95\% confidence interval 0.97-1.24), respectively (Table S2 and S3 in the ESM). This was consistent in the secondary analyses of these two populations (Table S2S6 in the ESM).

\section{Discussion}

In this post hoc exploratory analysis of the SUP-ICU trial, we found higher 90-day mortality and a lower percentage of days alive without use of life support in ICU patients at risk of GI bleeding with baseline SAPS II > 53 who were assigned to pantoprazole compared with those assigned to placebo in the primary complete-case population. These findings did not appear to be explained by baseline imbalance or differences in withdrawals or follow-up in this subgroup, but missing SAPS II values in the original trial cohort may have explained some of these findings.

We conducted this study because one of the predefined subgroup analyses in the SUP-ICU trial main analysis suggested higher 90-day mortality in the pantoprazole group among patients who had SAPS II $>53$ points (test of interaction, p-value 0.049) [7]. We may have explained some of this sub-group effect as per the sensitivity analyses estimating the effect of missing data, but $8-10 \%$ relative risk increase in mortality with pantoprazol remains unexplained. This is likely to be important to patients, relatives and health care professionals.

Most findings were consistent with the findings of the analyses of the full trial population, as neither GI bleeding, infections nor myocardial ischemia explained the potential subgroup effect.

To our knowledge it is rare to observe a potentially heterogenous intervention effect depending on baseline 


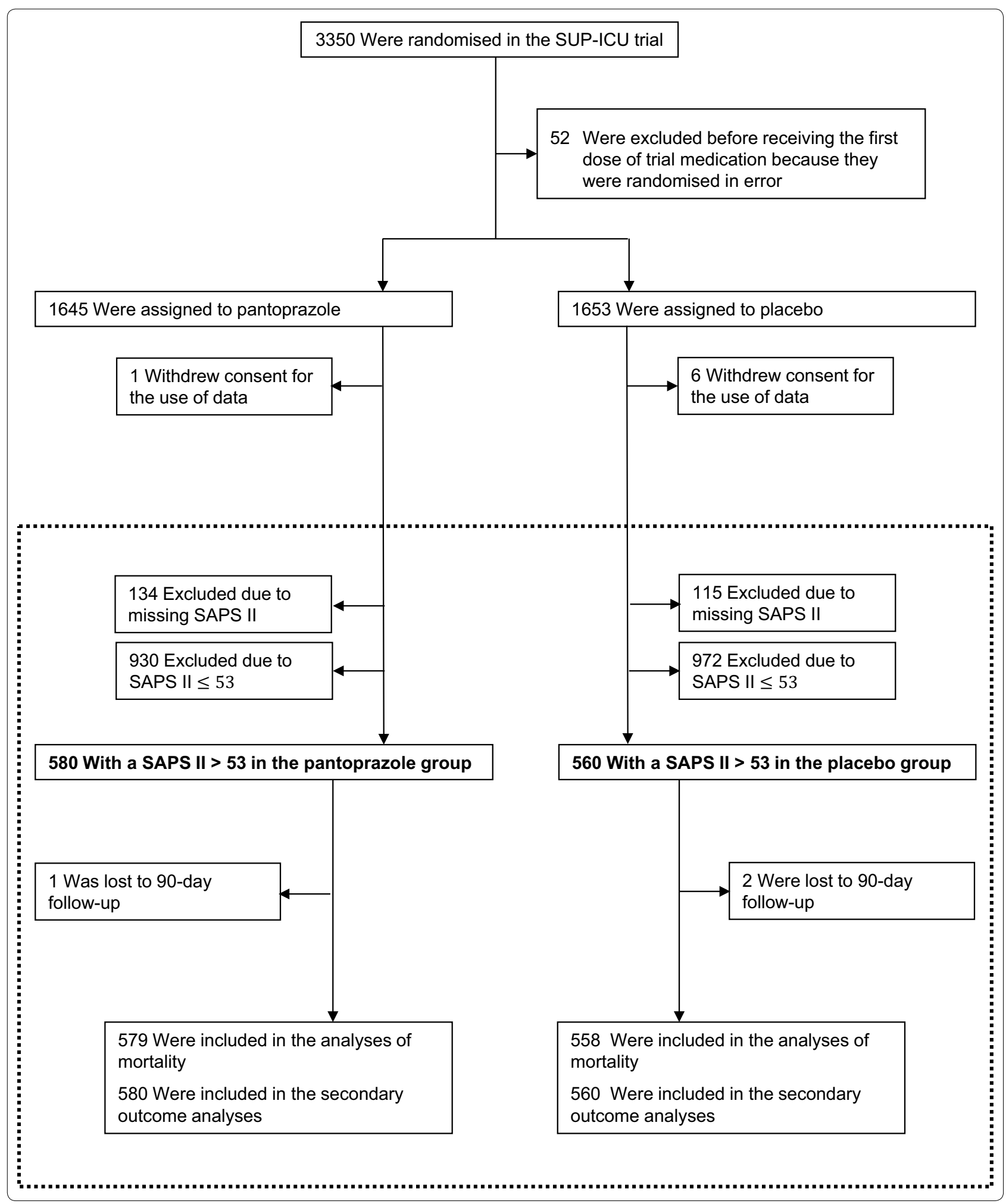


Table 1 Baseline characteristics of trial patients with baseline SAPS II > 53 in the complete-case population

\begin{tabular}{|c|c|c|}
\hline Characteristic & $\begin{array}{l}\text { Pantoprazole } \\
(N=580)\end{array}$ & $\begin{array}{l}\text { Placebo } \\
(N=560)\end{array}$ \\
\hline Age, years & $71(62-77)$ & $71(61-77)$ \\
\hline Male, no. (\%) & $357(62)$ & $371(66)$ \\
\hline \multicolumn{3}{|l|}{ Co-morbidities, no. (\%) } \\
\hline Chronic lung disease $^{\mathrm{a}}$ & $124(21)$ & $113(20)$ \\
\hline Previous myocardial infarction & $58(10)$ & $61(11)$ \\
\hline Chronic heart failure (NYHA III-IV) ${ }^{b}$ & $38(7)$ & $37(7)$ \\
\hline Use of glucocorticoids $s^{c}$ & $15(3)$ & $10(2)$ \\
\hline Hematological malignancy ${ }^{d}$ & $39(7)$ & $32(6)$ \\
\hline Metastatic cancer ${ }^{\mathrm{e}}$ & $27(5)$ & $29(5)$ \\
\hline $\operatorname{AIDS}^{f}$ & $4(1)$ & $1(0.2)$ \\
\hline Coagulopathy ${ }^{9}$ & $160(28)$ & $111(20)$ \\
\hline Admitted to university hospital, no. (\%) & $415(72)$ & $405(72)$ \\
\hline $\begin{array}{l}\text { Hours from ICU admission to randomisa- } \\
\text { tion }\end{array}$ & $13(5-23)$ & $13(5-21)$ \\
\hline $\begin{array}{l}\text { Days from hospital admission to randomi- } \\
\text { sation }\end{array}$ & $1(1-3)$ & $1(1-3)$ \\
\hline \multicolumn{3}{|l|}{ ICU admission type, no. (\%) } \\
\hline Medical & $386(67)$ & $357(64)$ \\
\hline Emergency surgery & $160(28)$ & $175(31)$ \\
\hline Elective surgery & $34(6)$ & $28(5)$ \\
\hline Use of invasive mechanical ventilation & $485(84)$ & $467(83)$ \\
\hline Use of vasopressors or inotropes & $436(75)$ & $417(74)$ \\
\hline Use of acute renal replacement therapy & $80(14)$ & $65(12)$ \\
\hline SAPS $\|^{h}$ & $63(58-72)$ & $63(58-71)$ \\
\hline SOFA score ${ }^{i}$ & $11(9-13)$ & $11(9-12)$ \\
\hline
\end{tabular}

Values with ranges are medians (interquartile ranges). A full list of risk factors for GI bleeding is provided in the ESM. There were no statistically significant differences between the groups in any baseline characteristic except for coagulopathy

a Chronic lung disease was defined as any history of chronic obstructive pulmonary disease, asthma or other chronic lung disease or treatment with any relevant drug indicating this at admission to hospital

b Chronic heart failure was defined as New York Heart Association Functional Class (NYHA) III-IV. NYHA III: the patient has marked limitations in physical activity due to symptoms (fatigue, palpitation or dyspnea) even during less than ordinary activity (walking short distances 20-100 m or walking up stairs to 1 st floor). The patient is only comfortable at rest. NYHA IV: the patient is not able to carry out any physical activity without discomfort (fatigue, palpitation or dyspnea). Symptoms are present even at rest and the patient is mostly bedbound

c Use of corticosteroids was defined as patients treated with at least $0.3 \mathrm{mg} / \mathrm{kg} /$ day of prednisolone equivalent for at least 1 month in the 6 months prior to ICU admission

d Hematological malignancy includes any of the following: acute lymphoblastic leukemia, acute myelogenous leukemia, chronic myelogenous leukemia, chronic lymphocytic leukemia, Hodgkin's disease, Non-Hodgkin lymphoma (e.g., small lymphocytic lymphoma, diffuse large B cell lymphoma, follicular lymphoma, mantle cell lymphoma, hairy cell leukemia, marginal zone lymphoma, Burkitt's lymphoma, post-transplant lymphoproliferative disorder, T cell prolymphocytic leukemia, B cell prolymphocytic leukemia, Waldenström's macroglobulinemia, other NK- or T cell lymphomas) and multiple myeloma/plasma cell myeloma

e Metastatic cancer: proven metastasis by surgery, computed tomography (CT) scan or any other method

f Acquired immune deficiency syndrome (AIDS) was defined as HIV positive patients with one or more AIDS defining diseases such as Pneumocystis jirovecci
Table 1 (continued)

pneumonia, Kaposi's sarcoma, lymphoma, tuberculosis or toxoplasma infection

9 Coagulopathy included both acute coagulopathy defined as platelets $<50 \times 10^{9} /$ I or international normalised ratio $>1.5$ or prothrombin time $>20 \mathrm{~s}$ at ICU admission and history of coagulopathy defined as coagulopathy within 6 months prior to hospital admission

h In the $24 \mathrm{~h}$ prior to randomisation. The Simplified Acute Physiology Score (SAPS) II [6] is calculated from 17 variables and ranges from 0 to 163 with higher scores indicating higher severity of disease (SAPS II table available in the ESM)

${ }^{\mathrm{i}}$ In the $24 \mathrm{~h}$ prior to randomisation. The sepsis-related organ failure assessment (SOFA) [27] score grades organ failure with sub-scoring ranging from 0 to 4 for each of six organ systems (cerebral, circulation, lungs, liver, kidney and coagulation). The aggregated score ranges from 0 to 24 with higher scores indicating more severe organ failure (SOFA score table available in the ESM)

severity of disease in ICU trials [12], and this has not previously been suggested (or investigated) for pantoprazole [13-16]. A priori, it could be speculated that patients with the highest severity of disease are at the greatest risk of adverse events and/or tolerate side effects poorly, e.g., due to more frailty, or organ failure, or perhaps these patients constitute a different phenotype to patients with less severity of disease. An association between use of proton pump inhibitors and cardiovascular events, e.g., myocardial ischemia and stroke has previously been suggested [17-19], however, a causal relation, and adverse cardiovascular effects of short-term use in high disease severity ICU patients remains unconfirmed.

More patients had coagulopathy at baseline in the pantoprazole group, however this did not affect the risk estimate of mortality in the included post hoc sensitivity analysis or the risk of clinically important GI bleeding (Table 2).

In general, subgroup analyses of randomised clinical trials are prone to type 1 and 2 errors and are therefore often misleading [20,21], and criteria to evaluate the credibility of subgroup analyses have been suggested [22, 23]. According to these criteria, the following aspects weaken the case for the suggested subgroup finding: the randomisation was not stratified for SAPS II being greater than 53 respectively lesser than or equal to 53 , inconsistency between our hypothesised subgroup effect direction (as pre-specified in the trial protocol [1]) and the actually observed effect direction [7], and the observed lower limit of the $95 \%$ confidence interval close to 1.00 (imprecision) [23].

The limitations of this study include the post hoc design resulting in increased risk of attrition and detection bias [24]. However, the analyses were conducted in accordance with the predefined analyses of the main trial population [8]. As in the analyses of the full trial population, we excluded patients with missing SAPS II in the primary analysis of this study. Patients with SAPS II $>53$ constituted only around a third of the full SUP-ICU trial population with resulting lower statistical power 
and increased risk of type 1 and 2 errors. The cut-off of SAPS II at 53 points can be debated. This cut-off represents a predicted mortality rate of $50 \%$ in the original model from 1993 [6], however, this is no longer the case due to improved ICU survival rates. Presumably it would now represent a predicted mortality of around $25 \%$ [25]. Whether other cut-offs would have yielded different results is unknown. Also, baseline imbalance may have affected results, even though additionally adjusting for coagulopathy did not change the estimates. Finally, we refrained from using multiple imputation to impute the missing SAPS II elements for sensitivity analysis as the large number of resulting populations with variations in sizes for subsequent analyses would be nearly impossible to account for and communicate clearly.

The strengths of this study include the fact that it is based on a large randomised trial with low risk of bias and that analyses were conducted according to the original statistical analysis plan, both reducing the risk of selection bias.

A recent editorial in the New England Journal of Medicine suggested that proton pump inhibitor prophylaxis may benefit the sickest ICU patients [26]. We do believe that our findings warrant further assessment and replication in randomised trials and meta-analyses with low risk of bias. We may also learn more from the pre-planned

\section{Table 2 Primary and secondary outcome measures in the complete-case population}

\begin{tabular}{|c|c|c|c|}
\hline Outcomes & Pantoprazole & Placebo & Relative risk $(95 \% \mathrm{CI})^{\mathrm{a}, \mathrm{b}}$ \\
\hline \multicolumn{4}{|l|}{ Primary outcome no./total no. (\%) } \\
\hline \multicolumn{4}{|l|}{ Primary analysis of death by day 90} \\
\hline Primary analysis ${ }^{c}$ & $272 / 579(47.0)$ & 229/558 (41.0) & $1.13(1.00-1.29)$ \\
\hline \multicolumn{4}{|l|}{ Secondary analyses of death by day 90} \\
\hline Additionally adjusted analysis ${ }^{d}$ & $270 / 576(46.9)$ & $228 / 557(40.9)$ & $1.14(0.99-1.30)$ \\
\hline Per-protocol analysis ${ }^{\mathrm{e}}$ & $227 / 488(46.5)$ & 189/475 (39.8) & $1.17(1.01-1.37)$ \\
\hline \multicolumn{4}{|l|}{ Secondary outcomes (primary analyses $\left.{ }^{\mathrm{c}}\right)^{\mathrm{f}}$ no./total no. (\%) } \\
\hline $\begin{array}{l}\text { One or more clinically important events (clinically important gastrointestinal bleed- } \\
\text { ing, pneumonia, Clostridium difficile infection or myocardial ischemia) }\end{array}$ & $146 / 580(25.2)$ & $147 / 560(26.3)$ & $0.93(0.75-1.16)$ \\
\hline One or more episodes of clinically important gastrointestinal bleeding & 19/580 (3.3) & $34 / 560(6.1)$ & $0.49(0.29-0.86)$ \\
\hline One or more infectious adverse events (pneumonia or Clostridium difficile infection) & 109/580 (18.8) & $109 / 560(19.5)$ & $0.94(0.73-1.21)$ \\
\hline Severe adverse reactions ${ }^{9}$ & $0 / 580(0.0)$ & $0 / 560(0.0)$ & - \\
\hline \multicolumn{4}{|l|}{ Median percentage of days (interquartile range) ${ }^{h}$} \\
\hline Alive without the use of life support ${ }^{i}$ & $81(9-95)$ & $85(36-96)$ & \\
\hline
\end{tabular}

$\mathrm{Cl}$ confidence interval, ICU intensive care unit

a Confidence intervals are not adjusted for the comparisons of multiple outcomes. $P$ values are not reported for the same reason (except for days alive without the use of life support, see footnote')

b Logistic regression analyses adjusted for the stratification variables (site and hematologic malignancy)

c The primary analyses were adjusted for the stratification variables (hematological malignancy and site)

d The secondary analyses were adjusted for stratification and design variables (age, type of admission (medical, elective surgery or emergency surgery), sepsis-related organ failure assessment (SOFA) score). Contrary to the full population, less than $5 \%$ had missing SOFA score in this population (likely due to overlap in patients with missing SAPS II (=>excluded) and SOFA score). We therefore do not report the multiple imputation analysis

e The definition of the per-protocol population is presented in the ESM

f Additionally adjusted (secondary) analyses of the secondary outcomes show similar results as the primary analyses and are therefore not presented

9 Defined as anaphylactic reactions, agranulocytosis, pancytopenia, acute hepatic failure, Steven-Johnson syndrome, toxic epidermal necrolysis, interstitial nephritis or angioedema (Quincke's edema) related to the intervention as judged by the treating clinicians and investigators

h Calculated as the number of days without use of invasive or non-invasive mechanical ventilation, infusion of vasopressor or inotropic agents or any form of renal replacement therapy divided by the number of days alive within the 90-day follow-up period

${ }^{i}$ Days alive without life support were analysed by the van Elteren test adjusted for site. $P$ value $=0.02$

\section{(See figure on next page.)}

Fig. 2 Time to death and relative risk of death at day 90 in the complete-case population. Part A shows the survival curves censored at day 90 for the two groups in the intention-to-treat population. Part B shows relative risks (RRs) with 95\% confidence intervals (CI) for the primary outcome measure of death at day 90 in the pantoprazole group compared with the placebo group in all patients with complete SAPS II>53 and in the five additional pre-defined subgroups assessed by logistic regression analysis adjusted for stratification variables. For the definition of shock, coagulopathy, and history of liver disease, at baseline, please see the ESM (inclusion criteria). Note that the coagulopathy subgroup included both acute coagulopathy and history of coagulopathy. Medical admission was when no surgery was performed for at least 1 week prior to ICU admission 
Part A - Time to death

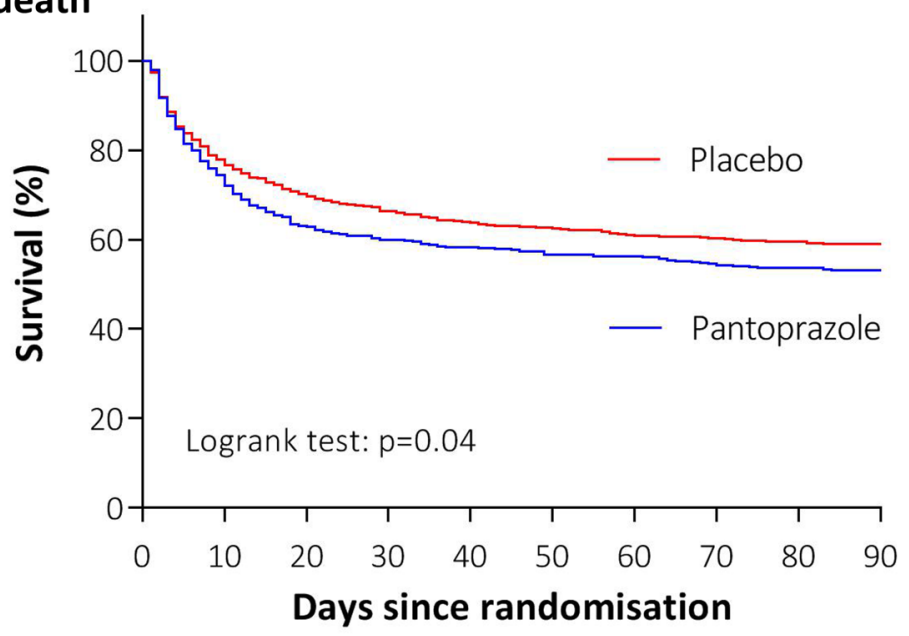

Number at risk

Pantoprazole

579

349

325

307

Placebo

558

375

341

\section{Part B - Relative risk of the primary outcome}

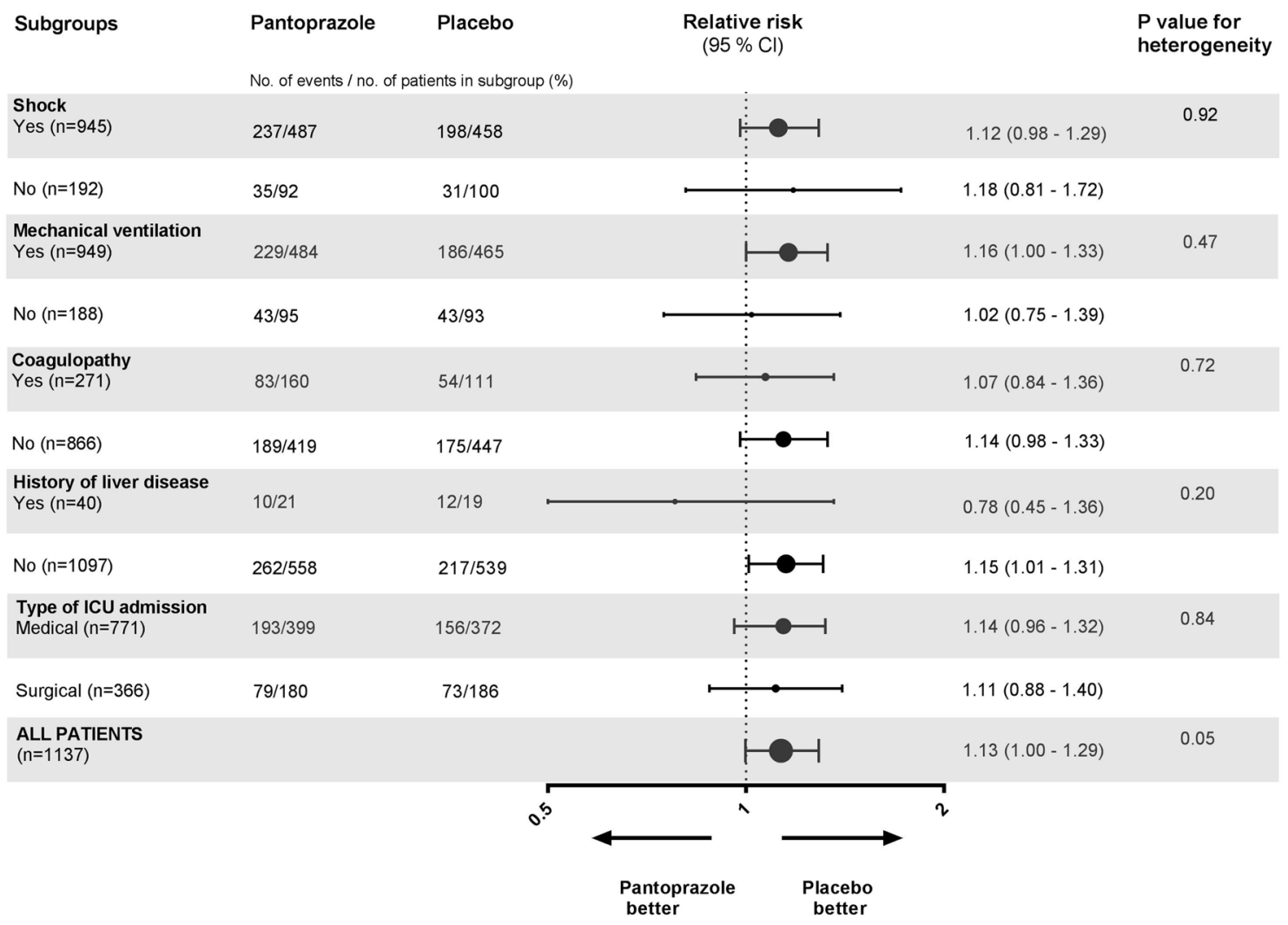


analyses of 1-year mortality after randomisation in all SUP-ICU trial patients and in the subgroups [1].

In conclusion, we found higher 90-day mortality and a lower percentage of days alive without the use of life support in the primary analysis in this post hoc investigation of ICU patients at risk of GI bleeding with complete baseline SAPS II > 53 assigned to pantoprazole versus placebo. Some of this may have been explained by missing SAPS II data in the full SUP-ICU trial cohort, but further research is needed to draw firm conclusions.

\section{Electronic supplementary material}

The online version of this article (https://doi.org/10.1007/s00134-019-05589-y) contains supplementary material, which is available to authorized users.

\begin{abstract}
Author details
1 Department of Intensive Care 4131, Copenhagen University Hospital, Rigshospitalet, Blegdamsvej 9, 2100 Copenhagen, Denmark. ${ }^{2}$ Centre for Research in Intensive Care (CRIC), Copenhagen University Hospital, Rigshospitalet, Copenhagen, Denmark. ${ }^{3}$ Copenhagen Trial Unit, Centre for Clinical Intervention Research, Copenhagen University Hospital, Rigshospitalet, Copenhagen, Denmark. ${ }^{4}$ Section of Biostatistics, University of Copenhagen, Copenhagen, Denmark. ${ }^{5}$ Center for Statistical Science, Peking University, Beijing, China. ${ }^{6}$ Department of Adult Critical Care, University Hospital of Wales, Cardiff, UK. ${ }^{7}$ Pharmacy Department, Oxford University Hospitals NHS Foundation Trust, Oxford, UK. ${ }^{8}$ Department of Intensive Care Medicine, Kuopio University Hospital, Kuopio, Finland. ${ }^{9}$ Department of Critical Care, University Medical Center Groningen, University of Groningen, Groningen, The Netherlands. ${ }^{10}$ Department of Anaesthesia and Intensive Care, Haukeland University Hospital, Bergen, Norway. ${ }^{11}$ Department of Clinical Medicine, UiB, Bergen, Norway. 12 Department of Intensive Care Medicine, Inselspital, Bern University Hospital, University of Bern, Bern, Switzerland.
\end{abstract}

\section{Acknowledgements}

All authors were involved in the conduct of the SUP-ICU trial. The authors thank everybody involved in the SUP-ICU trial: research staff and investigators, clinical staff, patients and their relatives.

\section{Funding}

The authors have received no specific funding for this study. The SUP-ICU trial was funded by Innovation Fund Denmark (4108-00011A) and supported by Rigshospitalet, the Capital Region of Denmark, the Regions of Denmark, the Scandinavian Society of Anaesthesiology and Intensive Care Medicine, Ehrenreich's Foundation, Aase and Ejnar Danielsens Foundation, the Danish Society of Anaesthesiology and Intensive Care Medicine, the Danish Medical Association and the European Society of Intensive Care Medicine. The Department of Intensive Care at Rigshospitalet has received support for other research projects from Fresenius Kabi, Germany; CSL Behring, Switzerland; Ferring Pharmaceuticals, Denmark; and the Novo Nordisk Foundation, Denmark. The SUP-ICU trial was funded by Innovation Fund Denmark and others.

\section{Compliance with ethical standards}

\section{Conflicts of interest}

None for the present study. The Department of Intensive Care at Rigshospitalet receives support for other research projects from Fresenius Kabi, Germany; CSL Behring, Switzerland; Ferring Pharmaceuticals, Denmark; and the Novo Nordisk Foundation, Denmark. The authors report no conflicts of interest. All authors were involved in the conduct of the SUP-ICU trial.

\section{Ethical approval}

No additional approvals were needed for this post hoc sub-analysis of the SUP-ICU trial. All procedures in the SUP-ICU trial were performed in accordance with the ethical standards of the institutional and/or national research committees and with the 1964 Helsinki Declaration and its later amendments or comparable ethical standards.

\section{Publisher's Note}

Springer Nature remains neutral with regard to jurisdictional claims in published maps and institutional affiliations.

Received: 20 November 2018 Accepted: 28 February 2019

Published online: 12 March 2019

\section{References}

1. Krag M, Perner A, Wetterslev J et al (2016) Stress ulcer prophylaxis with a proton pump inhibitor versus placebo in critically ill patients (SUP-ICU trial): study protocol for a randomised controlled trial. Trials 17:205

2. Krag M, Perner A, Wetterslev J et al (2015) Prevalence and outcome of gastrointestinal bleeding and use of acid suppressants in acutely ill adult intensive care patients. Intensive Care Med 41:833-845

3. Cook DJ, Griffith LE, Walter SD et al (2001) The attributable mortality and length of intensive care unit stay of clinically important gastrointestinal bleeding in critically ill patients. Crit Care 5:368-375

4. Rhodes A, Evans LE, Alhazzani W et al (2017) Surviving sepsis campaign: international guidelines for management of sepsis and septic shock: 2016. Intensive Care Med 43:304-377

5. Krag M, Perner A, Wetterslev J et al (2015) Stress ulcer prophylaxis in the intensive care unit: an international survey of 97 units in 11 countries. Acta Anaesthesiol Scand 59:576-585

6. Le Gall J-R, Lemeshow S, Saulnier F (1993) A new simplified acute physiology score (SAPS II) based on a European/North American Multicenter Study. JAMA 270:2957

7. Krag M, Marker S, Perner A et al (2018) Pantoprazole in patients at risk for gastrointestinal bleeding in the ICU. N Engl J Med 379:2199-2208

8. Krag M, Perner A, Wetterslev J et al (2017) Stress ulcer prophylaxis in the intensive care unit trial: detailed statistical analysis plan. Acta Anaesthesiol Scand 61:859-868

9. von Elm E, Egger M, Altman DG et al (2007) Strengthening the reporting of observational studies in epidemiology (STROBE) statement: guidelines for reporting observational studies. BMJ 335:806-808

10. Holst LB, Haase N, Wetterslev J et al (2014) Lower versus higher hemoglobin threshold for transfusion in septic shock. N Engl J Med 371:1381-1391

11. Jakobsen JC, Tamborrino M, Winkel P et al (2015) Count data analysis in randomised clinical trials. J Biomet Biostat 6:227

12. Macias WL, Nelson DR, Williams M et al (2005) Lack of evidence for qualitative treatment by disease severity interactions in clinical studies of severe sepsis. Crit Care 9:R607-R622

13. Alhazzani W, Guyatt G, Alshahrani M et al (2017) Withholding pantoprazole for stress ulcer prophylaxis in critically ill patients: a pilot randomized clinical trial and meta-analysis. Crit Care Med 45:1121-1129

14. Alhazzani W, Alshamsi F, Belley-Cote E et al (2018) Efficacy and safety of stress ulcer prophylaxis in critically ill patients: a network meta-analysis of randomized trials. Intensive Care Med 44:1-11

15. Cook D, Guyatt G (2018) Prophylaxis against upper gastrointestinal bleeding in hospitalized patients. N Engl J Med 378:2506-2516

16. Selvanderan SP, Summers MJ, Finnis ME et al (2016) Pantoprazole or placebo for stress ulcer prophylaxis (POP-UP). Crit Care Med 44:1842-1850

17. MacLaren R, Reynolds PM, Allen RR (2014) Histamine-2 receptor antagonists vs proton pump inhibitors on gastrointestinal tract hemorrhage and infectious complications in the intensive care unit. JAMA Intern Med 174:564-574

18. Charlot M, Ahlehoff O, Norgaard ML et al (2010) Proton-pump inhibitors are associated with increased cardiovascular risk independent of clopidogrel use: a nationwide cohort study. Ann Intern Med 153:378-386

19. Sehested TSG, Gerds TA, Fosbøl EL et al (2018) Long-term use of proton pump inhibitors, dose-response relationship, and associated risk of ischemic stroke and myocardial infarction. J Intern Med 283:268-281

20. Sedgwick P (2014) Randomised controlled trials: subgroup analyses. BMJ 349:g7513 
21. Wang R, Lagakos SW, Ware JH et al (2007) Statistics in medicine-reporting of subgroup analyses in clinical trials. N Engl J Med 357:2189-2194

22. Oxman AD, Guyatt GH (1992) A consumer's guide to subgroup analyses. Ann Intern Med 116:78-84

23. Sun X, Briel M, Walter SD, Guyatt GH (2010) Is a subgroup effect believable? Updating criteria to evaluate the credibility of subgroup analyses. BMJ 340:850-854

24. Higgins JPT, Altman DG, Gotzsche PC et al (2011) The Cochrane collaboration's tool for assessing risk of bias in randomised trials. BMJ 343:d5928

25. Granholm A, Møller MH, Krag M et al (2016) Predictive performance of the simplified acute physiology score (SAPS) II and the initial sequential organ failure assessment (SOFA) score in acutely ill intensive care patients: post-hoc analyses of the SUP-ICU inception cohort study. PLoS One 11:e0168948

26. Barkun A, Bardou M (2018) Proton-pump inhibitor prophylaxis in the ICU-benefits worth the risks? N Engl J Med 379:2263-2264

27. Vincent JL, Moreno R, Takala J et al (1996) The SOFA (sepsis-related organ failure assessment) score to describe organ dysfunction/failure. Intensive Care Med 22:707-710 